\title{
Cooperative communication between cognitive and primary users
}

\author{
Wei Liang, Soon Xin Ng, Lajos Hanzo \\ School of ECS, University of Southampton, SO17 1BJ, UK

Q1 Abstract: The active cooperation between a primary user (PU) and a cognitive user (CU) has the potential of leading to a transmission power reduction and transmission rate increase for both the PU and the CU. Alternatively, the required bandwidth may be reduced and the freed bandwidth may be leased to a group of CUs for their secondary communications. More explicitly, the authors cooperative protocol allows a CU to serve as a relay node (RN) for relaying the signal of the first

25 PU, which is a source node, to the second PU, which is a destination node (DN). Furthermore, the authors conceived adaptive turbo trellis-coded modulation (ATTCM) for appropriately adjusting both the code rate and the modulation mode according to the near-instantaneous channel conditions. The ATTCM switching thresholds specifically adjusted for ensuring that the bit error ratio is below $10^{-5}$ in order to minimise the potential error propagation from the RN to the DN. It was found that the joint design of coding, modulation, user-cooperation and cognitive radio (CR) techniques may lead to

30 significant mutual benefits for both the PUs and the CUs. More specifically, the authors propose an ATTCM aided two-way relaying cooperative $\mathrm{CR}$ scheme that maximises the CU's own data rate and improves the exploitation of the bandwidth released by the PUs. Their numerical and simulation results show that the bandwidth reduction attained by the proposed two-way relay-based CR scheme is more than $80 \%$ of the PU's bandwidth.

\section{Introduction}

40 Cognitive radio (CR), relying on a software-defined radio, is an emerging technology that enables the flexible development, construction, production, shipping and deployment of highly adaptive radios [1]. The two primary CR objectives defined in Haykin's paper [2] are

- Highly reliable communication whenever and wherever needed.

- Efficient utilisation of the radio spectrum.

50 The CR mechanism is also capable of exploiting the available spectrum holes in the communication spectrum. If the spectrum is not used by the primary users (PUs), then the cognitive users (CUs) would have the opportunity to access it for their secondary communications based on the $\mathrm{CR}$

55 technique. According to the $\mathrm{CR}$ protocol, the device listens to the wireless channel and identifies the spectrum holes, either in the time or in the frequency domain [1, 3, 4]. Moreover, the most common paradigms associated with CRs are the so-called underlay, overlay and interweave 60 networks. In the underlay paradigm, the CUs communicates with the aid of the PUs under the constraint that the interference imposed by the CUs on the PUs must not degrade the PUs' communication quality. In contrast to the underlay scheme, the CUs in the interwave paradigm can

65 only transmit simultaneously with a PU in the event of a false spectral hole detection. Thus in effect, the CU's transmit power is limited by the sensing-range of its spectral hole sensing, not by the interference experienced. Moreover, in the overlay paradigm, both the CU and PU communicates using the same frequency band in the same geographic space, assuming that the CUs assisted the PUs transmissions invoking cooperative communication techniques, such as advanced coding or cognitive relaying techniques $[5,6]$.

Cooperative communication [7] is a new communication paradigm that promises significant capacity and multiplexing gain improvement in wireless networks. It is capable of supporting users by providing an improved integrity or throughput with the advent of user cooperation [8]. The two most popular collaborative protocols are the decode-and-forward (DAF) and the amplify-and-forward (AAF) schemes [9]. Cooperative communication aided CR systems may be categorised into the following three types: (i) cooperation among the PUs; (ii) cooperation between PUs and CUs; and (iii) cooperation among the CU peers [10]. More specifically, the first type is similar to traditional cooperative communication. In the third type, a CU may act as a relay node $(\mathrm{RN})$ for other CUs, which may have different available spectra [10]. For the second type, the PUs have a higher priority than the CUs, where the CUs may act as RNs for PUs [11, 12]. More specifically, the active cooperation [11] among the PUs and CUs would allow the PUs to transmit at a lower power and/or at a higher throughput, whereas at the same time enabling the CUs to communicate using the released bandwidth. Another 
interesting protocol involving simultaneous transmissions of the PUs and CUs has been proposed in [4] for maximising 135 the overall achievable rate. In our work, we consider a cooperative $\mathrm{CR}$ scheme, which relies on cooperation of the source PU and the destination PU with the aid of the CUs acting as RNs. This is commonly referred to as the overlay paradigm, and various papers have focused on this model, when appointing a single PU [13, 14]. However, in these scenarios the PU's improved performance does not necessarily translate into a satisfactory performance for the CUs. In some cases, the CUs have limited spectrum access opportunities, if the PUs have their own data to transmit

145 [14]. In our approach, we aim for increasing the CU's own data rate by exploiting the bandwidth released by the PUs, as well as increasing the throughput of PUs by using one of these CUs as a RN. In our proposed one-way relay aided cooperative CR system, we have considered multiple CUs and a single PU. We have employed the relay selection technique of [15] for choosing the best CU to act as a RN in order to help the PU to successfully deliver its information. Moreover, we have also proposed a novel two-way relay aided cooperative $\mathrm{CR}$ scheme, which has

155 two PUs in the system. We have considered two protocols in this scenario. The first protocol is based on a time division broadcast channel (TDBC) [16], which relies on three time slots. The second protocol is based on a multiple-access broadcast channel (MABC) [16], which

160 requires only two time slots. In this contribution, we design coding and modulation schemes for an active cooperation-based CR system.

In CR systems the link-quality varies across a wide range, which cannot be adjusted by power-control. Hence, near-instantaneously adaptive coded modulation is proposed, which is capable of accommodating these differences. We have considered the idealistic adaptive schemes based on both the continuous-input continuous-output memoryless channel (CCMC) and on the 170 discrete-input continuous-output memoryless channel (DCMC) [17]. More specifically, the CCMC-based adaptive scheme assumes that idealistic capacity-achieving coding and modulation schemes are employed for communicating exactly at Shannon's capacity. By contrast, the

175 DCMC-based adaptive scheme assumes that an idealistic capacity-achieving code is employed for aiding the PSK/

Q2 QAM modulation schemes considered for the sake of operating right at the modulation-dependent DCMC capacity. Furthermore, we also considered a practical adaptive scheme based on power- and bandwidth-efficient turbo trellis-coded modulation (TTCM) [18], which is a joint coding and modulation scheme that has a structure similar to binary turbo codes. In addition, the TTCM schemes [19] were designed based on the best component trellis-coded modulation [20] components using the so-called 'punctured' minimal distance criterion for communicating over the additive white Gaussian noise channel. The transmission rate/throughput [or information bit-per-symbol (iBPS)] of our system is adapted according

190 to the instantaneous channel conditions. A higher-throughput TTCM scheme is employed when the channel conditions are good, whereas a lower-throughput TTCM scheme or no transmission is used, when the channel conditions are poor.

The novel contributions of our paper are

- The CUs' data rate is maximised by our proposed cooperative CR scheme. Similarly, the PU is also capable of transmitting at an improved transmission rate at a given SNR, whereas releasing a significant amount of its bandwidth for exploitation by the CUs.

- We conceive a two-way relaying scheme for our proposed CR system based on both the TBDC and the MABC protocols, which aims for jointly improving the power efficiency, the achievable rate and the throughput of PUs.

- A practical adaptive coded modulation scheme is investigated and benchmarked against idealistic adaptive schemes.

The rest of the paper is organised as follows. The system design of the idealistic cooperative CR scheme capable of operating at exactly Shannon's capacity is outlined in Section 2.1. The fixed modulation-based transmission model of our cooperative CR scheme is detailed in Section 2.2, whereas our realistic ATTCM-aided one-way relaying system is described in Section 2.3.2. The details of our ATTCM-aided two-way relaying assisted cooperative CR schemes are provided in Section 2.3.3. The overall performance of our proposed schemes is evaluated in Section 3, whereas our conclusions are presented in Section 4.

\section{System model}

\subsection{System design of our idealistic cooperative CR 225 scheme}

In this section, we adopt the cooperative CR philosophy of $[11,12]$ relying on the cooperation between a PU [as the source node ( $\mathrm{SN})$ ] and a $\mathrm{CU}$ (as the $\mathrm{RN}$ ) for conveying the source message to another PU [as the destination node (DN)]. To facilitate efficient spectrum sharing between the PU and CUs, we consider configuring and sharing the frequency bands of $W_{1}$ and $W_{2}$, as shown in Fig. 1. Observe in Fig. 1 that the CU acts as a RN and assists the $\mathrm{PU}$ in transmitting its signal in one of the frequency bands, namely in $W_{1}$. In the other frequency band, namely $W_{2}$, the PU remains silent and the CUs transmit their own signals by using the entire time slot $T$.

Again, Fig. 1 illustrates the bandwidth, time period and power allocation for the PU and CUs, where $T$ and $W_{0}$ are the original time period and bandwidth allocated for the $\mathrm{PU} / \mathrm{SN}$ [We represent the PU acting as the SN as PU/SN. Q3 Similarly, CU/RN denotes the CU acting as a RN, whereas $\mathrm{PU} / \mathrm{DN}$ denotes the PU acting as the DN.] to transmit its source message to the PU/DN. When the PU/SN is assisted by a $\mathrm{CU} / \mathrm{RN}$, the PU/SN only has to utilise a fraction of $T$ and $W_{0}$ in order to convey the source message to the $\mathrm{PU} / \mathrm{DN}$. More specifically, the $\mathrm{PU} / \mathrm{SN}$ and $\mathrm{CU} / \mathrm{RN}$ will share the bandwidth $W_{1}$ to convey the source message to the PU/DN, whereas the other CUs may use the remaining bandwidth of $\left(W_{2}=W_{0}-W_{1}\right)$ for their own communications. In other words, a CU/RN assists in saving some of the transmission power of the PU/SN because of the reduction of the transmission period from $T$ to $T_{1}$. In return, the PU/SN would release the bandwidth $W_{2}$ to other CUs. More specifically, let us assume that the transmission power per unit frequency emanating from the $\mathrm{PU} / \mathrm{SN}$ is $P_{\mathrm{S}}$ watts $/ \mathrm{Hz}$ and the target transmission rate is $R_{\mathrm{pu}}$ bits/s. The PU/SN transmits using the power of $P_{\mathrm{S}}$ during $T_{1}$, whereas the CU/RN forwards the source message using the power of $P_{\mathrm{CR}, 1}$ during $T_{2}$ and the second $\mathrm{CU}$ can broadcast its message to other CUs using the power of $P_{\mathrm{CR}, 2}$ during the entire time period $T$. 

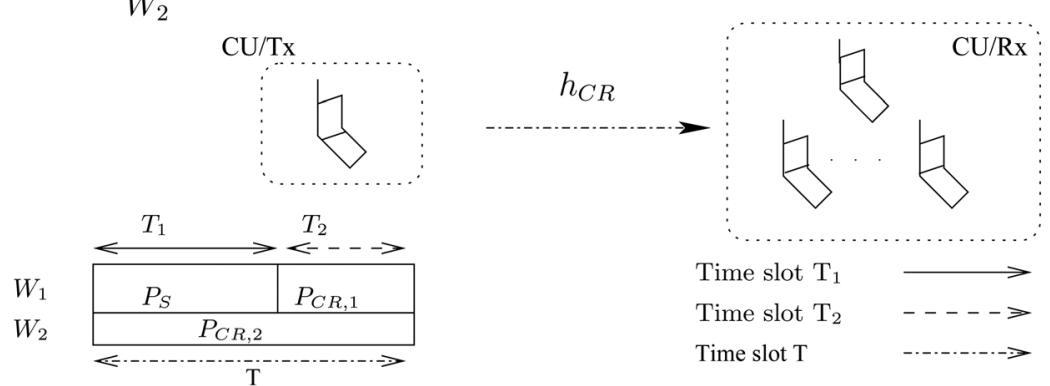

Tx: Transmiter Rx: Receiver

Fig. 1 PU's and CU's spectrum-access model

The bandwidth, time period and power allocation for the PU and CU The total time slot duration is $T=T_{1}+T_{2}$ and the bandwidth is $W_{0}=W_{1}+W_{2}$

During the first time slot $T_{1}$, the PU/SN broadcasts the source message $x$ to both the CU/RN and the PU/DN. The signal received at the PU/DN via the source-to-destination (SD) [The SD link is represented by the link between the $\mathrm{PU} / \mathrm{SN}$ and the PU/DN. The SR link represents the communication link between the PU/SN and the CU/RN. In addition, the communication link between the CU/RN and the $\mathrm{PU} / \mathrm{DN}$ is referred to as the RD link.] link is given by

$$
y_{\mathrm{sd}}=\sqrt{P_{\mathrm{S}}} h_{\mathrm{sd}} x+n_{\mathrm{sd}}
$$

and the signal received at the $\mathrm{CU} / \mathrm{RN}$ via source-to-relay (SR) link is

$$
y_{\mathrm{sr}}=\sqrt{P_{\mathrm{S}}} h_{\mathrm{sr}} x+n_{\mathrm{sr}}
$$

where $n_{\mathrm{sd}}$ and $n_{\mathrm{sr}}$ are the AWGN processes having an average single-sided noise power per unit frequency of $N_{0}=4.0 \times$ $10^{-21}$ watts/Hz [11] in the SD and SR links, respectively. In this contribution, we have adopted the AAF model of [11] and additionally we extended it to our DAF model. Hence, our CU/RN is capable of caring out either the AAF or the

\section{DAF operation.}

During the second time slot $T_{2}$ the CU/RN would forward the source message to the PU/DN using the transmission power of $P_{\mathrm{CR}, 1}$ watts/Hz. When considering the DAF protocol, provided that the RN is capable of decoding the transmitted symbol correctly, it forwards the decoded symbol with a power $P_{\mathrm{CR}, 1}$ to the DN. Otherwise the RN remains idle. The signal received by the PU/DN via the relay-to-destination (RD) link may be formulated as

$$
y_{\mathrm{rd}}^{\mathrm{DAF}}=\sqrt{P_{\mathrm{CR}, 1}} h_{\mathrm{rd}} x+n_{\mathrm{rd}}
$$

Similarly, when considering the AAF cooperation protocol, the CU/RN amplifies the received signal and forwards it to

received by the PU/DN via the RD link may be expressed as

$$
y_{\mathrm{rd}}^{\mathrm{AAF}}=\omega_{A} \sqrt{P_{\mathrm{CR}, 1}} h_{\mathrm{rd}} y_{\mathrm{sr}}+n_{\mathrm{rd}}
$$

where

$$
\omega_{A}=\frac{1}{\sqrt{P_{S}\left|h_{\mathrm{sr}}\right|^{2}+N_{0}}}
$$

is the amplification factor [21]. Then the signal received by the PU/DN under the AAF protocol via the RD link may be rewritten as

$$
\begin{aligned}
y_{\mathrm{rd}}^{\mathrm{AAF}} & =\frac{\sqrt{P_{\mathrm{CR}, 1}} h_{\mathrm{rd}} y_{\mathrm{sr}}}{\sqrt{P_{S}\left|h_{\mathrm{sr}}\right|^{2}+N_{0}}}+n_{\mathrm{rd}} \\
& =\frac{\sqrt{P_{\mathrm{CR}, 1} P_{S}} h_{\mathrm{rd}} h_{\mathrm{sr}}}{\sqrt{P_{S}\left|h_{\mathrm{sr}}\right|^{2}+N_{0}}} x+\frac{\sqrt{P_{\mathrm{CR}, 1}} h_{\mathrm{rd}}}{\sqrt{P_{S}\left|h_{\mathrm{sr}}\right|^{2}+N_{0}}} n_{\mathrm{sr}}+n_{\mathrm{rd}}
\end{aligned}
$$

The channel gains $h_{\mathrm{sd}}, h_{\mathrm{sr}}$ and $h_{\mathrm{rd}}$ are assumed to be independent complex Gaussian random variables with zero mean and variances of $\sigma_{\mathrm{sd}}^{2}, \sigma_{\mathrm{sr}}^{2}$ and $\sigma_{\mathrm{rd}}^{2}$, respectively. The channel variance is $[21,22]$

$$
\sigma_{a b}=\left(\frac{\lambda}{4 d_{a b} \pi}\right)^{\alpha}=\left(\frac{c}{4 d_{a b} f_{c} \pi}\right)^{\alpha}
$$

where $d_{a b}$ denotes the geometrical distance between node $a$ and node $b$, the wavelength is $\lambda=\left(c / f_{\mathrm{c}}\right)$, where $c$ is the speed of light and we consider a carrier frequency of $f_{\mathrm{c}}=350 \mathrm{MHz}$. Furthermore, we consider an outdoor environment, where the path-loss exponent [23] is given by $\alpha=3$.

In our scheme, the PU/SN transmits during $T_{1}$, whereas the $\mathrm{CU} / \mathrm{RN}$ transmits during $T_{2}$. Both the PU/SN and CU/RN utilise the bandwidth $W_{1}$. When the AAF protocol is 
employed based on Shannon's capacity theorem, the CCMC capacity of the cooperative relay channel over the bandwidth of $W_{1} \mathrm{~Hz}$ is given by

$$
C_{\mathrm{PU}}^{\mathrm{AAF}}=\frac{W_{1}}{2} \log _{2}\left[1+\frac{P_{\mathrm{S}}\left|h_{\mathrm{sd}}\right|^{2}}{N_{0}}+f_{\mathrm{CR}}\right]
$$

where we have [21, p. 122]

$$
f_{\mathrm{CR}}=\frac{P_{\mathrm{S}} P_{\mathrm{CR}, 1}\left|h_{s r}\right|^{2}\left|h_{\mathrm{rd}}\right|^{2}}{\left(P_{\mathrm{S}}\left|h_{\mathrm{sr}}\right|^{2}+P_{\mathrm{CR}, 1}\left|h_{\mathrm{rd}}\right|^{2}+N_{0}\right) N_{0}}
$$

When we consider the DAF protocol, the capacity of our system is limited by the capacity of either the SR link or that of the combined channel constituted by the SD and RD links which ever is lower. Then the CCMC capacity of DAF transmissions over $W_{1} \mathrm{~Hz}$ can be formulated as [21, p. 126]

$$
\begin{aligned}
C_{\mathrm{PU}}^{\mathrm{DAF}}= & \frac{W_{1}}{2} \min \left[\log _{2}\left(1+\frac{P_{\mathrm{S}}\left|h_{\mathrm{sd}}\right|^{2}}{N_{0}}+\frac{P_{\mathrm{CR}, 1}\left|h_{\mathrm{rd}}\right|^{2}}{N_{0}}\right),\right. \\
& \left.\times \log _{2}\left(1+\frac{P_{\mathrm{S}}\left|h_{\mathrm{sr}}\right|^{2}}{N_{0}}\right)\right]
\end{aligned}
$$

The factor (1/2) in (6) and (7) indicates that the PU only utilises the first time slot $T_{1}$ of Fig. 1, which the CU uses the second time slot $T_{2}$ to transmit its signals. Without loss of generality, we assume $T_{1}=T_{2}=(T / 2)$. Based on (6), the bandwidth required for achieving a transmission rate of $R_{\mathrm{PU}} \leq C_{\mathrm{PU}}^{\mathrm{AAF}}$ may be formulated as (see (8))

For the DAF protocol, the bandwidth requirement of $W_{1}$ can be expressed as (see (9))

In the non-cooperative case, the CCMC capacity of the PU/ $\mathrm{SN}$ is given by

$$
C_{\mathrm{PU}}^{*}=W_{0} \log _{2}\left[1+\frac{P_{\mathrm{PU}}\left|h_{\mathrm{sd}}\right|^{2}}{N_{0}}\right]
$$

For the following derivation, we use the $C_{\mathrm{PU}}$ to represent $C_{\mathrm{PU}}^{\mathrm{DAF}}$ and $C_{\mathrm{PU}}^{\mathrm{AAF}}$. It can be shown that the transmission power originally required for achieving $R_{\mathrm{PU}}=C_{\mathrm{PU}}$ is given by

$$
P_{\mathrm{PU}}=\frac{N_{0}\left(2^{\left(R_{\mathrm{PU}} / W_{0}\right)}-1\right)}{\left|h_{\mathrm{sd}}\right|^{2}}
$$

As seen in Fig. 1, a group of CUs is capable of communicating using the released bandwidth $W_{2}$ for the entire period of $T$, whereas a $\mathrm{CU}$ is helping the PU/SN as a $\mathrm{RN}$. The received signal for CUs to transmit its own signal in the whole time slot $T$ is given by

$$
y_{\mathrm{CR}}=\sqrt{P_{\mathrm{CR}, 2}} h_{\mathrm{CR}} x_{\mathrm{CR}}+n_{\mathrm{CR}}
$$

where $h_{\mathrm{CR}}$ denotes the channel between a CU's transmitter $(\mathrm{CU} / \mathrm{SN})$ and its destination $(\mathrm{CU} / \mathrm{DN})$ for its own transmission. The source message $x_{\mathrm{CR}}$ transmit from $\mathrm{CU} / \mathrm{SN}$ to $\mathrm{CU} / \mathrm{DN}$ and $n_{\mathrm{CR}}$ is the AWGN process. Then, the achievable transmission rate of the CUs is given by

$$
R_{\mathrm{CR}}=W_{2} \log _{2}\left[1+\frac{P_{\mathrm{CR}, 2}\left|h_{\mathrm{CR}}\right|^{2}}{N_{0}}\right]
$$

If the total transmission power of CUs is limited to $P_{\mathrm{CR}}$, then we have

$$
P_{\mathrm{CR}}=\frac{1}{2} P_{\mathrm{CR}, 1} W_{1}+P_{\mathrm{CR}, 2} W_{2}
$$

In this way, the CUs can decide how to allocate their joint transmission power in order to maximise their own data rate. Let us define the ratio of transmission power allocated for helping the $\mathrm{PU} / \mathrm{SN}$ to the total transmission power of the CUs over the bandwidth $W_{1}$ as

$$
\psi=\frac{(1 / 2) P_{\mathrm{CR}, 1} W_{1}}{P_{\mathrm{CR}}}
$$

where $\psi=\left[\begin{array}{ll}0 & 1\end{array}\right]$. Similarly, the ratio of the transmission power allocated to transmit the CUs' data to the total transmission power of the CUs, over the bandwidth $W_{2}$ can be defined as

$$
1-\psi=\frac{P_{\mathrm{CR}, 2} W_{2}}{P_{\mathrm{CR}}}
$$

More specifically, the transmission power $P_{\mathrm{CR}, 1}$ at $\mathrm{CU} / \mathrm{RN}$ may be determined from (9) and (15). On the other hand, the CU's own data rate using the released bandwidth $W_{2}=$ $W_{0}-W_{1}$ may be derived as

$$
R_{\mathrm{CR}}=\left(W_{0}-W_{1}\right) \log _{2}\left[1+\frac{P_{\mathrm{CR}}\left|h_{\mathrm{CR}}\right|^{2}(1-\psi)}{\left(W_{0}-W_{1}\right) N_{0}}\right]
$$

which can be optimised with respect to $\psi$. Moreover, the reduced-distance-related-pathloss-reduction (RDRPR) [24, 25 ] experienced in our system by the SD, SR and RD links with respect to the SD link as a benefit of its reduced distance-based path-loss can be expressed as [24]

$$
G_{\mathrm{sd}}=\left(\frac{d_{\mathrm{sd}}}{d_{\mathrm{sd}}}\right)^{3}, \quad G_{\mathrm{sr}}=\left(\frac{d_{\mathrm{sd}}}{d_{\mathrm{sr}}}\right)^{3} \quad \text { and } \quad G_{\mathrm{rd}}=\left(\frac{d_{\mathrm{sd}}}{d_{\mathrm{rd}}}\right)^{3}
$$

respectively. Naturally, the RDRPR of the SD link with respect to itself is unity, that is, we have $G_{\text {sd }}=1$. Our

$$
W_{1} \geq \frac{2 R_{\mathrm{PU}}}{\log _{2}\left[1+\left(P_{\mathrm{S}}\left|h_{\mathrm{sd}}\right|^{2} /\left(N_{0}\right)\right)+\left(\left(P_{\mathrm{S}} P_{\mathrm{CR}, 1}\left|h_{\mathrm{sr}}\right|^{2}\left|h_{\mathrm{rd}}\right|^{2}\right) /\left(\left(P_{\mathrm{S}}\left|h_{\mathrm{sr}}\right|^{2}+P_{\mathrm{CR}, 1}\left|h_{\mathrm{rd}}\right|^{2}+N_{0}\right) N_{0}\right)\right)\right]}
$$

$$
W_{1} \geq \frac{2 R_{\mathrm{PU}}}{\min \left[\log _{2}\left(1+\left(P_{\mathrm{S}}\left|h_{\mathrm{sd}}\right|^{2} /\left(N_{0}\right)\right)+\left(P_{\mathrm{CR}, 1}\left|h_{\mathrm{rd}}\right|^{2} /\left(N_{0}\right)\right)\right), \quad \log _{2}\left(1+\left(P_{\mathrm{S}}\left|h_{\mathrm{sr}}\right|^{2} /\left(N_{0}\right)\right)\right)\right]}
$$




\subsection{Fixed-mode transmission in cooperative $C R$ schemes}

535 In this section, we investigate the achievable bandwidth reduction based on three fix-mode transmission schemes. More specifically, System $A$ in Fig. 2 is a non-cooperative system, whereas System $B$ and System $C$ are relay aided cooperative CR systems. We assume that both the SN and

540 the DN are PUs and the RN is a CU. The passband bandwidth $\zeta$ of PSK/QAM modulation is assumed to be the same as the Baud-rate (or symbol rate) of $R_{\mathrm{S}} \mathrm{symbol} / \mathrm{s}$, whereas the baseband bandwidth is given by $R_{\mathrm{S}} / 2 \mathrm{symbol} / \mathrm{s}$, when an ideal low-pass filter is assumed. The bit rate of the

545 system is $R_{\mathrm{b}}=\eta \times R_{\mathrm{s}}(\mathrm{bit} / \mathrm{s})$, where $\eta$ is the throughput in bit-per-symbol (bps). When considering a pathloss exponent of $\alpha=3$, we have a RDRPR of $G=2^{\alpha}=8$, which is $\tilde{G}=10 \log _{10}(8)=9 \mathrm{~dB}$ when the $\mathrm{RN}$ is located at the mid-point between the $\mathrm{SN}$ and the $\mathrm{DN}$. The received signal-to-noise ratio (SNR) $\left(\mathrm{SNR}_{r}\right)$ in decibel is given by

$$
\mathrm{SNR}_{r}=\mathrm{SNR}_{t}+\tilde{G}
$$

and the transmit SNR [The concept of transmit SNR [25] is 55 unconventional, as it relates quantities to each other at two physically different locations, namely the transmit power to the noise power at the receiver, which are at physically different locations.] is $\mathrm{SNR}_{t}=10 \log _{10}\left(P_{\mathrm{t}} / N_{0}\right)$, where $P_{\mathrm{t}}$ is the transmit power and $N_{0}$ is the single-sided noise power.

560 We assume that a BER of $10^{-5}$ or less is required at the $\mathrm{DN}$, where received SNRs of 9 and $18 \mathrm{dBs}$ are required at the DN, when TTCM-8PSK and TTCM-64QAM are employed, respectively. The SD link is assumed to be of low quality and hence it is not considered in this example.

As seen from Fig. 2, the PU/SN of System $B$ is capable of increasing its throughput to $\eta_{B}=2.5 \mathrm{bps}$ from the $\eta_{A}=2 \mathrm{bps}$

TTCM-8PSK: 2 bps

$$
\begin{aligned}
& \eta_{A}=2 \mathrm{bps} \\
& \zeta_{A}=R_{s}^{A} \mathrm{sym} / \mathrm{s} \\
& \mathrm{SNR}_{t}=9 \mathrm{~dB}
\end{aligned}
$$

\section{$\mathrm{S}$}

TTCM-64QAM: 5 bps

$\eta_{B}=2.5 \mathrm{bps}$

$\zeta_{B}=R_{s}^{A} \mathrm{sym} / \mathrm{s}$

580

$\mathrm{SNR}_{t}=9 \mathrm{~dB}$

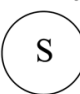
$\tilde{G}=9 \mathrm{~dB}$

System A

BER $<10^{-6}$

$R_{b}^{A}=2 R_{s}^{A} \mathrm{bit} / \mathrm{s}$

$\mathrm{SNR}_{r}=9 \mathrm{~dB}$

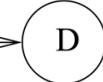

System B

BER $<10^{-6}$

$R_{b}^{B}=2.5 R_{s}^{A} \mathrm{bit} / \mathrm{s}$

$\mathrm{SNR}_{r}=18 \mathrm{~dB}$

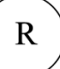

TTCM-64QAM: 5 bps

$\eta_{C}=2.5 \mathrm{bps}$

$\zeta_{C}=0.8 R_{s}^{A} \mathrm{sym} / \mathrm{s}$

$\mathrm{SNR}_{t}=9 \mathrm{~dB}$

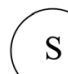

$\mathrm{S}$ $\tilde{G}=9 \mathrm{~dB}$

stem C

BER $<10^{-6}$

$R_{b}^{C}=2 R_{s}^{A} \mathrm{bit} / \mathrm{s}$

590

Fig. 2 Comparison of a non-cooperative scheme and two relay-assisted DAF-CR schemes, where the target $S N R_{t}$ is $9 \mathrm{~dB}$ and the target BER is below $10^{-5}$ value of System $A$, when using the same bandwidth of $\zeta=$ $R_{\mathrm{s}}$. Their bit rate rate (BER) is $\left(R_{\mathrm{b}}^{A} / R_{\mathrm{b}}^{B}\right)=\left(2 R_{\mathrm{s}}^{A} / 2.5 R_{\mathrm{s}}^{B}\right)$ upon assuming that System $A$ and System $B$ have the same symbol rate of $R_{\mathrm{s}}^{A}=R_{\mathrm{s}}^{B}$, the relationship of their bit rate is given by

$$
R_{\mathrm{b}}^{B}=\frac{\eta_{B}}{\eta_{A}}, \quad R_{\mathrm{b}}^{A}=1.25 R_{\mathrm{b}}^{A}
$$

Thus, System $B$ has a $25 \%$ higher bit rate than System $A$ within the same bandwidth. Then the relationship between the bit rate of System $B$ and symbol rate of System $A$ is $R_{\mathrm{b}}^{B}=1.25 R_{\mathrm{b}}^{A}=1.25 \times 2 R_{\mathrm{s}}^{A}=2.5 R_{\mathrm{s}}^{A}$.

By contrast, both System $A$ and System $C$ have the same bit rate of $R_{\mathrm{b}}^{A}=R_{\mathrm{b}}^{C}$, whereas the relationship of their symbol rates is given by

$$
R_{\mathrm{s}}^{C}=\frac{\eta_{A} R_{\mathrm{s}}^{A}}{\eta_{C}}=0.8 R_{\mathrm{s}}^{A}
$$

Hence, System $C$ is capable of providing the same bit rate using only $80 \%$ of the original bandwidth. This is achieved at a lower Baud-rate of $p R_{\mathrm{s}}$, where $p=\left(\eta_{A} / \eta_{C}\right)=(2 / 2.5)=$ 0.8 is the throughput ratio of System $A$ to System $C$. Then the relationship between the bit rate of System $C$ and the symbol rate of System $A$ is $R_{\mathrm{b}}^{C}=2 R_{\mathrm{s}}^{A}$.

More specifically, the bandwidth-reduction factor is given by

$$
B_{\mathrm{s}}=1-\frac{\eta_{A}}{\eta_{C}}
$$

Therefore a CU assist in PU's transmission could save 20\% $(1-0.8=0.2=20 \%)$ of PU's bandwidth. Thus, this saved bandwidth can then be shared among other CUs. If we create a System $D$ in a practical approaches where the bit rate of $\mathrm{PU}$ is lower than that of System $B$, but higher than that of System $A$, then we have $R_{\mathrm{b}}^{D}=1.1 R_{\mathrm{b}}^{A}$. By referring to Fig. 2, we have $\eta_{A}=2.0$ bps and $\eta_{D}=2.5$ bps. Furthermore, we have $R_{\mathrm{b}}^{D}=1.1 \eta_{A} \times R_{\mathrm{s}}^{A}=1.1 \times 2 \times R_{\mathrm{s}}^{A}=2.2 R_{\mathrm{s}}^{A}$. Based on (20), we have $R_{\mathrm{s}}^{D}=\left(\eta_{A} R_{\mathrm{s}}^{A} / \eta_{D}\right)=(2.2 / 2.5) R_{\mathrm{s}}^{A}=$ $0.88 R_{\mathrm{s}}^{A}$. Then the bandwidth-reduction factor becomes $B_{\mathrm{s}}=$ $1-\left(\eta_{A} / \eta_{D}\right)=1-0.88=0.12$. In this situation, System $D$ is capable of reducing the original bandwidth by $12 \%$ for the CU's benefit, whereas the PU enjoys an additional 0.5 bps throughput increment.

\subsection{Practical ATTCM-aided cooperative CRs}

We have shown that it is possible and practical for the PU to release the available bandwidth for supporting the CU's own transmission in exchange for an increased transmission throughput based on the analysis of Section 2.2. In this section, ATTCM aided one-way and two-way relaying schemes are proposed for cooperative CR applications.

2.3.1 ATTCM algorithm: In our proposed system, we will make use of this power- and bandwidth-efficient TTCM scheme. Employing TTCM has the advantage that the system's effective throughput can be increased upon increasing the code rate, when the channel-quality improves. In addition, both the BER and frame error ratio performance of the system may be improved when TTCM is used [26]. Recently, various TTCM schemes were designed in [27] with the aid of the Extrinsic Information Transfer charts $[28,29]$ and union bounds for the sake of 


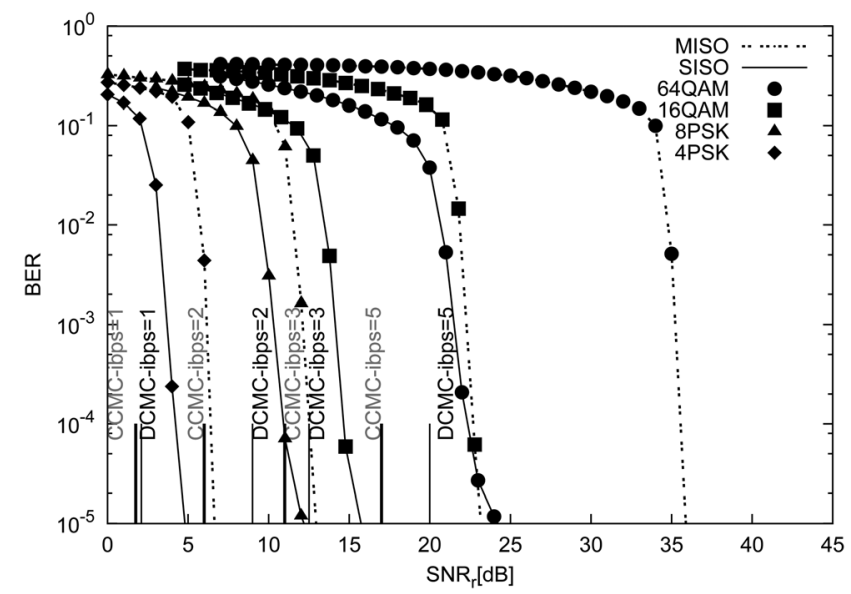

Fig. 3 BER against SNR $R_{r}$ performance of TTCM aided MISO system and TTCM aided SISO system

A frame length of 1200 symbols was employed when communicating over uncorrected Rayleigh channels

Four TTCM iterations and four outer iterations with the MISO detector is used in MISO system

approaching the capacity of the Rayleigh fading channel. The TTCM encoder comprises two identical parallelconcatenated TCM encoders [20] linked by a symbol interleaver. The first TCM encoder directly processes the original input bit sequence, whereas the second TCM encoder manipulates the interleaved version of the input bit sequence. Then the bit-to-symbol mapper maps the input bits to complex-valued symbols using the set partition (SP)-based labelling method [26]. The structure of the TTCM decoder is similar to that of binary turbo codes, but each decoder alternately processes its corresponding encoder's channel-impaired output symbol and then the other encoder's channel-impaired output symbol [26, p. 764]. More details on the TTCM principles may be found in [26]. We have employed a ATTCM scheme for protecting the SR and the RD links, where the effective throughput (or iBPS) range is given by $\mathrm{iBPS}=\{0,1,2,3$, $5\}$ bps when no transmission, QPSK, 8PSK, 16QAM and 64QAM are considered, respectively.

Moreover, the ATTCM mode switching thresholds $r=\left[\gamma_{0}\right.$, $\left.\gamma_{1}, \gamma_{2}, \gamma_{3}\right]$ are determined based on the BER performance curves of each of the four TTCM schemes communicating over Rayleigh channels, as shown in Fig. 3. Specifically, we consider the five TTCM modes and the ATTCM mode switching operation based on the following algorithm (see equation at the bottom of the page)

2.3.2 One-way relaying aided cooperative $C R$ scheme: As shown in Fig. 1, we consider a single SN, $K$ RNs and a single DN. All relays operate in the half-duplex DF mode and it is assumed that each relay only knows its own channel, but the DN receiver knows all channel value with the aid of training. In addition, the benefit of the direct SD link is also considered. The signal received by node $b$ from node $a$ is given by

$$
y_{a b}=\sqrt{G_{a b}} \sqrt{P_{a b}} h_{a b} x+n
$$

where $G_{a b}$ denotes the RDRPR experienced by the link between node $a$ and node $b$, whereas $h_{a b}$ represents the symmetric quasi-static Rayleigh fading channel gain of the $a b$ link and we assume that all channel gains are independent of each other. The quasi-static Rayleigh fading channels between the $\mathrm{SN}$ and the RNs are denoted as $\left\{h_{\mathrm{sr}_{k}}\right\}_{k=1}^{K}$, whereas those between the RNs and the DN are represented by $\left\{h_{r_{k} d}\right\}_{k=1}^{K}$. The power term $P_{a b}$ is normalised to unity. We use the notation $\gamma_{a b}$ to refer to the instantaneous receive SNR of the link between node $a$ and node $b$, so that

$$
\gamma_{a b}=\frac{G_{a b}\left|h_{a b}\right|^{2}}{N_{0}}
$$

Among these $K$ available relays only the specific relay with the highest instantaneous SNR is selected for forwarding the signal transmitted from the SN to the DN. The channel condition $\left|h_{s r_{i}}\right|,\left|h_{r_{i} d}\right|$, at each relay, which has included $G_{a b}$, describes the quality of the SR and RD links, when using the $i$ th RN. The capacity of this two-hop scheme is limited by the particular hop that has the minimum SNR or $\left\{\min \left[\left|h_{\mathrm{sr}}\right|^{2},\left|h_{r \cdot d}\right|^{2}\right]\right\}$, which is also referred to as 'bottleneck' [15]. We have $(K+1)$ links spanning from the SN to the DN supported by $K$ RNs as well as the SD link. We have considered the max-min relay selection technique for maximising the transmission rate, which relies on the policy defined as [30]

$$
k=\arg \max _{i}\left\{\min \left[\left|h_{\mathrm{sr}_{i}}\right|^{2},\left|h_{r_{i}}\right|^{2}\right]\right\}
$$

Under this policy, the best RN imposes the most restrictive 'bottleneck' among the RNs. We have considered a single-input-single-output (SISO) [31] system for both the $\mathrm{SR}$ and RD link in our one-way relay system, where each node has one antenna, and employed maximum-likelihood (ML) multiuser detection (MUD). The ML MUD provides the best possible performance at the cost of the highest complexity, which is a non-linear detector and it is optimal in terms of minimising the symbol error probability, when all possible vectors are equal likely [32]. In our proposed system, we have considered perfect channel estimation. In the non-cooperative scheme of System $A,\left|h_{\mathrm{sd}}\right|^{2}$ is used for computing the $\mathrm{SNR}_{r}$. In addition, all CRs are located near the center of the system, hence, we have $G_{\mathrm{sr}}=G_{\mathrm{rd}}=8$. As we discussed in Section 2.3.1, each of our communication links is assisted by the ATTCM scheme. As seen from Fig. 3, we specifically chose the switching thresholds for ensuring that the BER at the RN became lower than $10^{-5}$, which are given by $r_{\mathrm{ATTCM}}=[6.5,15.5,22,35.8] \mathrm{dB}$. We note that Shannon's CCMC capacity is only restricted by the SNR and the bandwidth. The CCMC-based (perfect-code-related) switching thresholds are represented

$$
\text { MODE }= \begin{cases}\gamma_{R}>\gamma_{3}, & \text { TTCM }-64 \mathrm{QAM}, \text { BPS }=5 \mathrm{bps} \\ \gamma_{2}<\gamma_{R} \leq \gamma_{3}, & \text { TTCM }-16 \mathrm{QAM}, \mathrm{BPS}=3 \mathrm{bps} \\ \gamma_{1}<\gamma_{R} \leq \gamma_{2}, & \text { TTCM }-8 \mathrm{PSK}, \mathrm{BPS}=2 \mathrm{bps} \\ \gamma_{0}<\gamma_{R} \leq \gamma_{1}, & \text { TTCM }-4 \mathrm{PSK}, \mathrm{BPS}=1 \mathrm{bps} \\ \gamma_{R} \leq \gamma_{0}, & \text { No }- \text { Tx, BPS }=0 \mathrm{bps}\end{cases}
$$


as $r_{\mathrm{CCMC}}=[1.75,6,11,14] \mathrm{dB}$, whereas the switching thresholds of the corresponding DCMC-based scheme are 795 given by $\Upsilon_{\mathrm{DCMD}}=[2,8,12.5,20] \mathrm{dB}$, which are also explicitly shown in Fig. 3.

Although the Systems $B, C$ and $D$ introduced in Section 2.2 are all relay-aided cooperative $\mathrm{CR}$ schemes, we have had different design objectives for their bit rate and symbol rate.

800 Hence, they also illustrate the different benefits of cooperative CR networks. Let us now refer to Figs. $5 a$ and $b$, where we consider ATTCM instead of fixed-mode 8PSK and 64QAM. The system labelled as ATTCM-System $A$ is the classic non-cooperative scheme, whereas 805 ATTCM-System $B$, ATTCM-System $C$ and ATTCMSystem $D$ are all relay-aided cooperative CR schemes. ATTCM-System $B$ achieves a higher bit rate by invoking user-cooperation within the same bandwidth, as ATTCM-System $A$. By contrast, ATTCM-System $C$ achieves

810 a higher bandwidth reduction by employing user-cooperation, whereas maintaining the same bit rate as ATTCM-System $A$. Furthermore, ATTCM-System $D$ achieved both a practical bit rate improvement as well as a bandwidth reduction. For the future implementation and 815 investigation, we could also use the other coding schemes including LDPC instead of TTCM.

\subsubsection{Two-way relaying aided cooperative $C R$} scheme: Having studied the ATTCM aided one-way relaying assisted cooperative CR scheme in Section 2.3.2, we will now consider how the one-way relay system may be extended to a two-way relaying assisted cooperative CR system, where the two PUs act as the SNs and the DNs for each other.

We have considered two protocols in our proposed scheme namely the TDBC and MABC. In the TDBC protocol shown in Fig. 4, there is no interference hence the corresponding complexity at the $\mathrm{RN}$ is kept low. Three time slots are used for two data flows, which are $s_{1} \rightarrow r, s_{2} \rightarrow r$ and $s_{1} \leftarrow r \rightarrow$ $s_{2}$, where $s_{1}$ and $s_{2}$ denote the two primary sources, whereas $r$ denotes the $\mathrm{CU}$ which acts as a RN. By contrast, the MABC protocol requires two time slots for transmitting two data flows, which are $s_{1} \rightarrow r \leftarrow s_{2}$ and $s_{1} \leftarrow r \rightarrow s_{2}$. Since the sources transmit their information simultaneously, the MABC system suffers from self-interference. In our paper, we have invoked an advanced MUD technique at the $\mathrm{RN}$ in order to decode both information streams of the SNs and to cancel the self-interference. Explicitly, in the MABC protocol, two signals were transmitted simultaneously from the two PU/SNs, where each PU has a single antenna. In addition, we have used the powerful ML MUD for

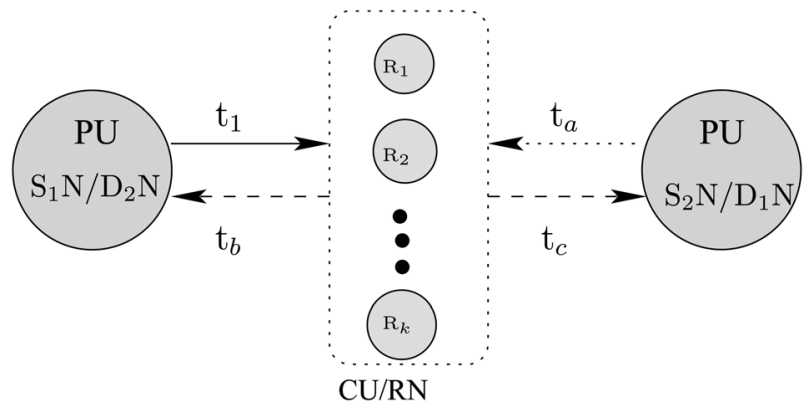

860

Fig. 4 Schematic of a two-way relay-aided system, including two $P U s$ and $K C U s$

For MABC, $t_{a}=t_{1}$ and $t_{b}=t_{c}=t_{2}$, where $t_{1}$ is the first transmission period and $t_{2}$ is the second transmission period. For TDBC, $t_{a}=t_{2}$ and $t_{b}=t_{c}=t_{3}$, where $t_{3}$ is the third transmission period

detecting the two source signals using a single-antenna aided CU/RN, which constitutes a $(2 \times 1)$-element multiple-input-single-output [31] system for the SR links. This powerful MUD was required for eliminating avalanche-like error propagation at the RNs. However, opted for $(1 \times 1)$-element SISO system for the RD link, where each DN employs a single antenna for detecting its wanted signal arriving from the $\mathrm{RN}$. By contrast, in the TDBC protocol, we have a $(1 \times 1)$-element SISO system in the two SR links and a single RD link, because the two SNs use two separate time periods for transmitting their information to the $\mathrm{RN}$, respectively.

We have opted for appointing the best relay has the set of available $K$ RNs that experience identically and independently distributed fading. Then the selected best RN decodes and forwards the received signals to the intended destinations, namely to the $\mathrm{PU} / \mathrm{DN}_{1}$ and $\mathrm{PU} / \mathrm{DN}_{2}$, respectively, during the second cooperative transmission period. Hence, the overall system throughput becomes higher than that of a one-way relaying scheme, which requires two time slots for transmitting a single user's information. Again, each of the communication links is assisted by our ATTCM scheme. By referring to (22), the signal transmitted from the $\mathrm{SNs}$ to the $k$ th $\mathrm{RN}$ in our MABC two-way relay system is given by

$$
y_{\mathrm{sr}_{k}}=\sqrt{G_{s r_{k}}} \sqrt{P_{s r_{k}}} h_{\mathrm{sr}_{k}} \boldsymbol{X}+\boldsymbol{n}
$$

where $\boldsymbol{X}$ is a vector hosting both SNs' signal. We can view the Q4 two SNs as a combined two-antenna assisted SN, where (24)
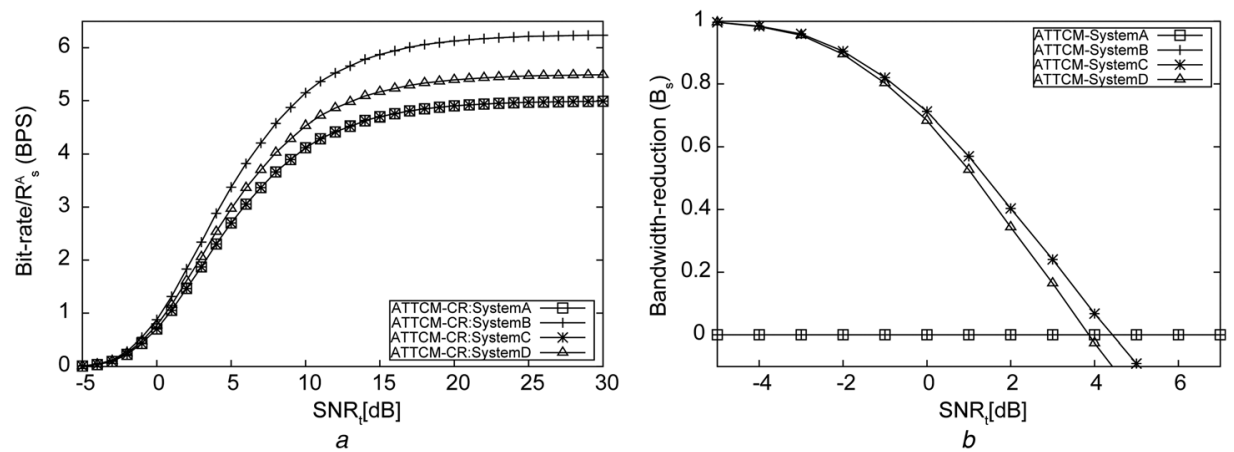

Fig. 5 Comparison of ATTCM aided System A, System B, System C and System D 
is a $(2 \times 1)$-element MISO system. Furthermore, the signal received by the DNs from the RNs is given by

$$
y_{r_{k} d}=\sqrt{G_{r_{k}} d} \sqrt{P_{r_{k} d}} h_{r_{k} d} x+n
$$

930 where the RDRPR experienced by each link is defined as $G_{\mathrm{SR}_{1}}=G_{\mathrm{SR}_{2}}=G_{\mathrm{RD}_{1}}=G_{\mathrm{RD}_{2}}=2^{3}=8$ in our system, since the RN is located midway between the $\mathrm{SN}$ and the DN. In addition, the channel gains $h_{S_{1} R_{k}}, h_{S_{2} R_{k}}$, $h_{R_{k} D_{1}}$ and $h_{R_{k} D_{2}}$ are independent of each other, each of which is represented by a quasi-static Rayleigh fading channel. The Gaussian noise vector $\boldsymbol{n}$ has a zero mean and a noise variance of $N_{0} / 2$ per dimension. By contrast, the SNR at the $k$ th SR link is computed as

$$
\gamma_{\left(r, \mathrm{sr}_{k}\right)}=\frac{G_{\mathrm{sr}_{k}}\left|h_{\mathrm{sr}_{k}}\right|^{2}}{N_{0}}
$$

Similarly, the SNR at the RD receiver is given by

$$
\gamma_{\left(r, r_{k} d\right)}=\frac{G_{r_{k} d}\left|h_{r_{k} d}\right|^{2}}{N_{0}}
$$

Again, we have considered the max-min relay selection technique as defined in (23) for selecting the best RN.

The ATTCM-TDBC-based switching thresholds were designed for ensuring that the BER at the $\mathrm{RN}$ becomes lower than $10^{-5}$, which are represented as $\Upsilon_{\mathrm{ATTCM}}^{\mathrm{TDBC}}=[6.5,15.5,22,35.8] \mathrm{dB}$, based on Fig. 3. In addition, as seen from Fig. 3, we have chosen the ATTCM-MABC switching thresholds for ensuring that the BER at the RN became lower than $10^{-5}$, which are given by: $Y_{\mathrm{ATTCM}}^{(\mathrm{MABC}, \mathrm{SR})}=[4.8,12,16,24] \mathrm{dB} \quad$ and $\quad \mathrm{Y}_{\mathrm{ATTCM}}^{(\mathrm{MABC}, \mathrm{RD})}=$ $[6.5,15.5,22,35.8] \mathrm{dB}$. The reason why we have chosen the BER at the RN to be lower than $10^{-5}$ for the MABC protocol is because the error floor emerging at $\mathrm{BER}<10^{-5}$ can be removed by using a long outer code, such as a Reed Solomon Code. The performance of the TDBC and the MABC aided schemes is characterised in Section 3.3.

\section{Performance results}

\subsection{Performance of idealistic cooperative CR schemes}

The relationship of the power ratio $\psi$ and the data rate of the $\mathrm{CU}$ is shown in Figs. $6 a$ and $b$. Fig. $6 a$ illustrates the CU's own data rate with respect to the power ratio $\psi$, when the RDRPR factors are given by $G_{\text {sd }}=G_{\mathrm{rd}}=1$ and $G_{\mathrm{sr}}$. We assume that the total bandwidth is $W_{0}=1 \mathrm{MHz}$ and the target transmission rate of the PU/SN is $R_{\mathrm{PU}}=500 \mathrm{kbits} / \mathrm{s}$. The total transmission power of the $\mathrm{CU}$ is $P_{\mathrm{CR}}=10 \mathrm{dBm}$. In this system, we assumed that the PU has maintained the same transmission power, which is $P_{\mathrm{S}}=P_{\mathrm{PU}}$ based on (11). Then, we plotted the data rate of the $\mathrm{CU}$ based on three different values of the distance $d_{\text {cr }}$ between the $\mathrm{CU}$ and its own destination, namely for $d_{\mathrm{cr}}=500 \mathrm{~m}, 1 \mathrm{~km}$ and $2 \mathrm{~km}$. Finally, the optimum ratios of the relay power over the total power budget are given by $64.5,53$ and $45 \%$ (with respect to $d_{\mathrm{cr}}=500 \mathrm{~m}, 1 \mathrm{~km}$ and $2 \mathrm{~km}$ ) when using the DAF protocol. Similarly, the optimum power ratios for the AAF protocol are given by 82,72 and $65 \%$. As seen in Fig. 5, a $\mathrm{CU} / \mathrm{RN}$ has offered a proportion of its transmission power to help the PU/SN, whereas $0 \leq \psi \leq 0.4$. During this period, the CU's own data rate is identical to zero. Fig. 5 shows the corresponding results when the $\mathrm{RN}$ is right in the middle of the PU/SN and PU/DN link, where the RDRPR factors are given by $G_{\text {sd }}=1$ and $G_{\text {sd }}=G_{\text {rd }}=8$. The optimum ratio of the relay power over the total power is 16,14 and $12 \%$ for $d_{\mathrm{cr}}=500 \mathrm{~m}, 1 \mathrm{~km}$ and $2 \mathrm{~km}$, respectively, when using DAF detection. Moreover, the corresponding values for AAF detection are given by 33,20 and $12 \%$.

Observe in Figs. $6 a$ and $b$ that if the CU/RN is half-way between the $\mathrm{SN}$ and the $\mathrm{DN}$, a $\mathrm{CU} / \mathrm{RN}$ only has to dedicate a smaller proportion of its transmission power for aiding the $\mathrm{PU} / \mathrm{SN}$. Furthermore, as $d_{\mathrm{cr}}$ increases, the CU's own data rate drops because its increased path loss.

\subsection{Performance of one-way relay aided cooperative $C R$ schemes}

Fig. 7 shows the iBPS against $\mathrm{SNR}_{t}$ performance of the ATTCM, CCMC and DCMC aided one-way relay schemes. As seen from Fig. 7, the curves recorded for the CCMC and DCMC modes are close to each other, when employing only one RN. For $\mathrm{SNR}_{t}>28 \mathrm{~B}$, the iBPS value of the three system schemes became saturated at 5 bps. In general, the CCMC-System $A$ and CCMC-System $C$ arrangements represent the upper bound, because the CCMC capacity quantifies the highest throughput. As seen in Fig. 7, the intersection point of the ATTCM-SystemC and ATTCM-System $A$ schemes is at $\mathrm{SNR}_{t}=8 \mathrm{~dB}$, whereas those for the CCMC-System $C$ and CCMC-System $A$ modes is at $1 \mathrm{~dB}$ and that for the DCMC mode is at $1.2 \mathrm{~dB}$. At their intersection point, the throughput of System $A$ and System $C$ are equal. The throughput of the 'CCMC mode' is always better than that of the DCMC and ATTCM

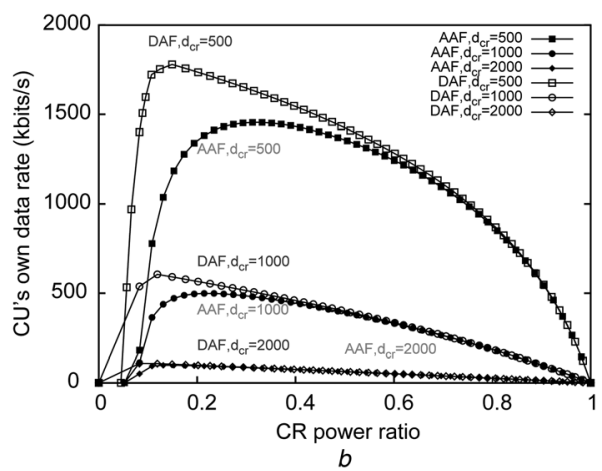

Fig. 6 CU's own data rate based on DAF and AAF detections 


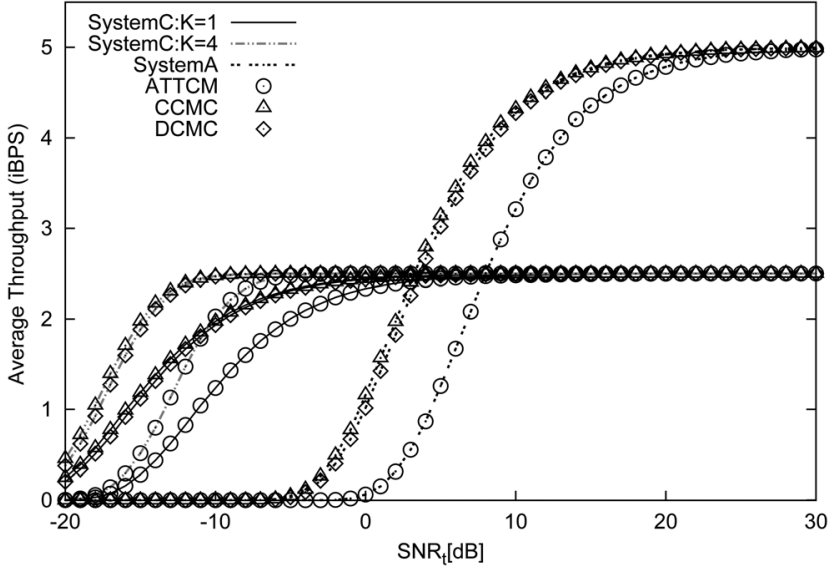

Fig. 7 iBPS against $S N R_{t}$ of the ATTCM, CCMC and DCMC aided $C R$ schemes when communicating over quasi-static Rayleigh fading

A BER below $10^{-5}$ is maintained and $G_{\mathrm{sr}}=G_{\mathrm{rd}}=8$. The 'SystemC' is represented by relay aided system of Section 2.3.2, whereas the 'System $A$ ' is refer to the non-cooperative System $A$ of Section 2.3.2. The number of relays is $K=1$ and $K=4$

schemes. In addition, the throughputs of all the System $C$ is higher than those of the System $A$ before their intersection point. Cooperation is no longer beneficial beyond the intersection point. Observe that when the number of RNs is increased to $K=4$, the System $C$-related curves converge to the asymptotic value of $2.5 \mathrm{bps}$ for $\mathrm{SNR}_{t}>-8 \mathrm{~dB}$, which is $10 \mathrm{dBs}$ earlier than their counterparts having $K=1$. This is because when the number of RNs is increased, we have a higher chance of selecting a better $\mathrm{RN}$ for assisting the PU/SN

Fig. 8 portrays the performance of the corresponding iBPS value against $\mathrm{SNR}_{t}$ of the ATTCM, CCMC and DCMC aided one-way relay in the cooperative $\mathrm{CR}$ schemes, when communicating over quasi-static Rayleigh fading channels, in comparison to that of Shannon's capacity bound. We will refer to the CCMC capacity-based curves as the upper

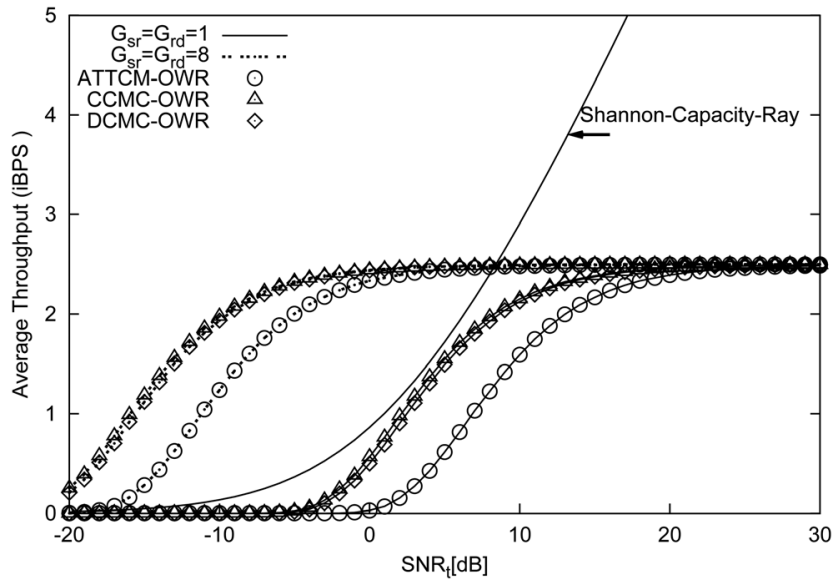

Fig. 8 iBPS against $S N R_{t}$ of the ATTCM, CCMC and DCMC aided one-way relay in $C R$ schemes when communicating over quasi-static Rayleigh fading channels compared to the CCMC capacity in Rayleigh channel

The 'Shannon-Capacity-Ray' label refers to the CCMC capacity in Rayleigh channel. The number of relays is $K=1$

The 'OWR' is represented by one-way relay system

The corresponding RDRPR are $G_{\mathrm{sr}}=G_{\mathrm{rd}}=8$ and $G_{\mathrm{sr}}=G_{\mathrm{rd}}=1$ bound. However, the DCMC capacity is more pertinent in the context of designing realistic channel-coded modulation schemes. Since we consider a RDRPR of $G_{\mathrm{sr}}=G_{\mathrm{rd}}=8$, the curves of the one-way relay aided cooperative $\mathrm{CR}$ schemes are not within Shannon's capacity bound given for the Rayleigh channel. In addition, the iBPS value of the CCMC, DCMC and ATTCM aided CR schemes saturated at $2.5 \mathrm{bps}$ for $\mathrm{SNR}_{t}=11 \mathrm{~dB}$. If the RDRPRs are changed to $G_{\mathrm{sr}}=G_{\mathrm{rd}}=1$, the resultant curves no longer exceed Shannon's capacity, where their iBPS curves saturated at $2.5 \mathrm{bps}$ for $\mathrm{SNR}_{t}=20 \mathrm{~dB}$, which were shifted to the right by about $9 \mathrm{~dB}\left(\log _{10}\left(2^{3}\right)=9 \mathrm{~dB}\right)$ with respect to those using $G_{\mathrm{s} r}=G_{\mathrm{rd}}=8$. There are two transmission links in our one-way scheme, namely the SR link and the RD link. Based on our ATTCM mode switching thresholds, the system will activate the 64QAM mode for $\gamma_{R}>\gamma_{3}$. If both the SR and RD links have achieved their best performance associated with 64QAM, then the throughput of these two links becomes $5 \mathrm{bps}$. We have two time slots in our cooperative $\mathrm{CR}$ scheme, where both the $\mathrm{SN}$ and $\mathrm{RN}$ transmitted the same amount of information. Thus, our overall system throughput becomes

$$
\eta_{\mathrm{SRD}}^{\text {one-way }}=\frac{\mathrm{iBPS}_{\mathrm{SR}}+\mathrm{iBPS}_{\mathrm{RD}}}{2 \times \text { Timeslot }}=\frac{5+5}{2 \times 2}=2.5
$$

BPS in the high-SNR region.

\subsection{Performance of two-way relay aided cooperative CR systems}

As seen in Fig. 9 , both our proposed ATTCM aided MABC and TDBC two-way relay system have a higher throughput than the proposed one-way relay system, when we consider the same number of RNs in the one-way relay system. In the MABC two-way relay system, the iBPS value became saturated at $5 \mathrm{bps}$ for $\mathrm{SNR}_{t} \geq 11 \mathrm{~dB}$. The two $\mathrm{SNs}$ send their information simultaneously to a $\mathrm{RN}$ and then the $\mathrm{RN}$ broadcasts the combined information to the two DNs. The system throughput of the MABC two-way relay system is

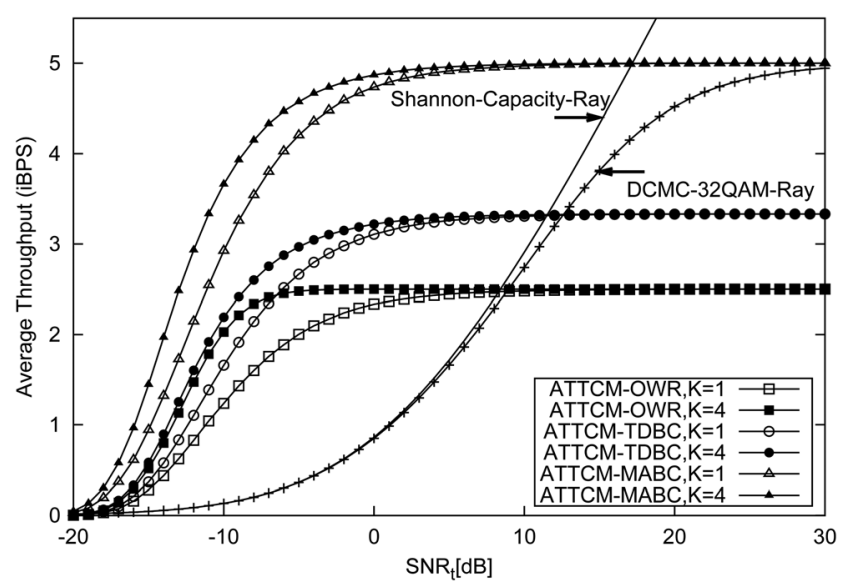

Fig. 9 iBPS against $S N R_{t}$ of the ATTCM aided one-way relay system and two-way relay system when communicating over quasi-static Rayleigh fading channels

The 'DCMC-32QAM-Ray' refers to the DCMC capacity for 32QAM modulation in Rayleigh channel and the 'Shannon-Capacity-Ray' refers to the CCMC capacity in Rayleigh channel

The 'OWR' is represented by one-way relay system

A BER below $10^{-5}$ is maintained and $G_{\mathrm{sr}}=G_{\text {rd }}=8$ 


$$
\eta_{\mathrm{SRD}}^{\mathrm{MABC}}=\frac{\mathrm{iBPS}_{S_{1} R}+\mathrm{iBPS}_{S_{2} R}+\mathrm{iBPS}_{R_{1} D}+\mathrm{iBPS}_{R_{2} D}}{2 \times \text { Timeslot }}
$$

When the MABC system has encountered the best possible channel conditions, the throughput became $\eta_{\mathrm{SRD}}^{\mathrm{MABC}}=(5+5+5+5 / 2 \times 2)=5 \mathrm{bps}$. Therefore the asymptotic throughput of our proposed MABC two-way relay system $\left(\eta_{\text {SRD }}^{\text {two-way }}\right)$ is twice that of the one-way relay scheme $\left(\eta_{\mathrm{SRD}}^{\text {one-way }}\right)$. By contrast, the iBPS value of the TDBC two-way relay system became saturated at $3.3 \mathrm{bps}$ for $\operatorname{SNR} t \geq 12 \mathrm{~dB}$. The TDBC two-way relay system required three time slots, hence its $\eta_{\mathrm{SRD}}$ value is lower than that of the MABC scheme beyond $\mathrm{SNR}_{t}=1 \mathrm{~dB}$. The throughput of the TDBC two-way relay system is $\eta_{\mathrm{SRD}}^{\mathrm{TDBC}}=(5+5+5+5 / 2 \times 3)=3.3 \mathrm{bps}$, when each link has achieved its best condition. Moreover, its asymptotic throughput is $0.8 \mathrm{bps}$ higher than the throughput of the one-way relay system. Fig. 9 shows the corresponding iBPS against $\mathrm{SNR}_{t}$ performance of our proposed two-way relay system in comparison to the CCMC and DCMC capacity. In addition, the curve of the MABC scheme are overlapped with the DCMC capacity for 32QAM at $\mathrm{SNR}_{t} \geq 30 \mathrm{~dB}$.

Fig. 10 illustrates the attainable bandwidth-reduction $\left(B_{\mathrm{s}}\right)$ against $\mathrm{SNR}_{t}$ for the ATTCM, the CCMC and the DCMC aided one-way relay as well as for the ATTCM-aided two-way relay schemes. As seen from Fig. 10, the attainable bandwidth-reduction $B_{\mathrm{s}}$ is slightly higher for the one-way relay scheme, when the number of RNs is increased from $K=1$ to $K=4$. It is also interesting to observe that the practical ATTCM scheme is capable of reducing the bandwidth more substantially compared to the idealistic DCMC and CCMC schemes. Furthermore, as the SNR increases, the bandwidth-reduction factor also reduces. This is because when the SNR is high, the quality of the SD link is sufficiently high for a fixed transmission throughput of $5 \mathrm{bps}$. The inclusion of a RN at high SNRs would only double the transmission period, without actually increasing the transmission throughput. Hence, we are only interested in the operational region, whereas we have $B_{\mathrm{s}}>0$. Note furthermore from Fig. 7 that at an SNR of $5 \mathrm{~dB}$, the ATTCM-System $A$ scheme can only achieve a throughput of

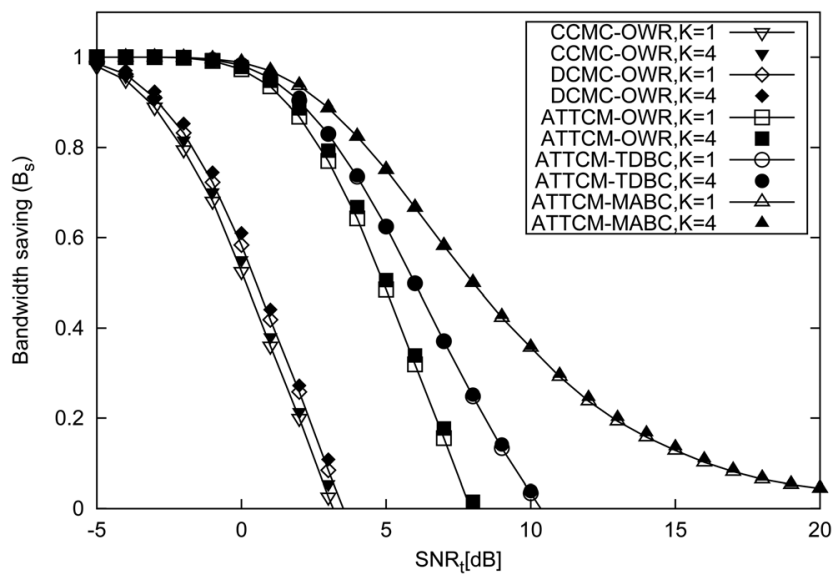

Fig. 10 Bandwidth reduction $B_{S}$ against $S N R_{t}$ for ATTCM aided one-way relay and two-way relay $C R$ schemes, when communicating over flat Rayleigh fading channels and maintaining a BER below $10^{-5}$

The number of frames simulated was 1000

The 'OWR' is represented by one-way relay system

$G_{\mathrm{sr}}=G_{\mathrm{rd}}=8$, the number of RNs in these schemes is $K=1$ and $K=4$
0.6 bps. However, with the aid of the best RN selected from four cooperating CUs, the ATTCM-System $C$ would enable the PU to transmit at a throughput of $2.48 \mathrm{bps}$. This may also be translated into a maximum achievable bandwidth reduction of $(1-(0.6 / 2.48))=0.758=75.8 \%$. Fig. 10 also illustrates the attainable bandwidth-reduction $\left(B_{\mathrm{S}}\right)$ against $\mathrm{SNR}_{t}$ for the ATTCM aided MABC and TDBC two-way relay system. Since we are only interested in the operational region of $B_{\mathrm{s}}>0$, for $\mathrm{SNR}_{t}>15 \mathrm{~dB}$, the proposed schemes relying on one-way relaying are no longer beneficial for the range of $B_{\mathrm{s}}<0$. The proposed two-way relaying scheme can use the entire bandwidth, since the bandwidth-reduction of the two-way relay scheme is always higher than zero. Observe from Fig. 7 that at an $\mathrm{SNR}_{t}$ of $5 \mathrm{~dB}$, the ATTCM-System $A$ scheme achieves a throughput of $0.6 \mathrm{bps}$. However, as seen in Fig. 10, the ATTCM-System $C$ regime relying on TDMC two-way relaying would enable the PU to transmit at a throughput of $3.2 \mathrm{bps}$. Similarly, the ATTCM-SystemC relying on MABC two-way relaying would enable the PU to transmit at a throughput of $3.9 \mathrm{bps}$. This may be translated into a bandwidth reduction of (1 $(0.6 / 3.2))=0.81=81 \%$ for the TDBC scheme. In addition, it will lead to a bandwidth reduction of $(1-(0.6 / 4.7))=$ $0.87=87 \%$ for the MABC scheme. Furthermore, the bandwidth reduction can be increased by $81-75.8=5.2 \%$ upon employing the TDBC scheme compared to the one-way relay scheme. In addition, $81-75.8=11.2 \%$ bandwidth reduction can be attained by employing the MABC scheme in comparison to the one-way relay system. Moreover, at a given SNR, the two-way relay-aided system always attains a higher $B_{\mathrm{s}}$ value, than the corresponding one-way relay system.

\section{Conclusions}

In this contribution, we have studied DAF and AAF assisted active cooperative $\mathrm{CR}$ schemes and quantified the optimum power ratio required for achieving the best transmission throughput for the CU. We proposed a practical ATTCM aided one-way relaying CR scheme, where adaptive coding and modulation were invoked according to the instantaneous channel conditions. We found that the proposed one-way $\mathrm{CR}$ scheme enables the PU to transmit at an improved transmission rate fora given SNR, whereas releasing a significant amount of bandwidth for exploitation by the CUs, despite operating at a reduced SNR. In the process of implementing the ATTCM aided one-way relaying CR scheme, we have also considered the max-min relay selection technique in order to choose the best relay for relaying the transmitted information from the SNs. Furthermore, in order to maximise the CU's own data rate and to improve the exploitation of the bandwidth released by the PUs, we also proposed a ATTCM aided two-way relay $\mathrm{CR}$ scheme by employing the MABC and TDBC protocols. The simulation results demonstrated that the two-way relay aided CR scheme is capable of achieving a higher bandwidth reduction then one-way relay aided CR scheme as well as simultaneously improving the system's average throughput.

\section{Acknowledgments}

The financial support of the EPSRC UK under the China-UK Science Bridge and the India-UK Advanced Technology Centre, as well as that of the European Union under the 
auspices of the CONERTO project is gratefully acknowledged. This work has been presented in part at the IEEE WCNC, Paris, France, April 2012.

\section{References}

1 Jondral, F.K.: 'Cognitive radio: a communications engineering view', IEEE Wirel. Commun. , 2007, 14, pp. 28-33

2 Haykin, S.: 'Cognitive radio: brain-empowered wireless communications', IEEE J. Sel. Areas Commun., 2005, 23, pp. 201-220

3 Mitola, J., Maguire, G.Q.: 'Cognitive radio: making software radios more personal', IEEE Pers. Commun., 1999, 6, pp. 13-18

4 Devroye, N., Mitran, P., Tarokh, V.: 'Achievable rates in cognitive radio channels', IEEE Trans. Inf. Theory, 2006, 52, pp. 1813-1827

5 Goldsmith, A., Jafar, S., Maric, I., Srinivasa, S.: 'Breaking spectrum gridlock with cognitive radios: an information theoretic perspective', Proc. IEEE, 2009, 97, pp. 894-914

6 Manna, R., Louie, R., Li, Y., Vucetic, B.: 'Cooperative spectrum sharing in cognitive radio networks with multiple antennas', IEEE Trans. Signal Process., 2011, 59, pp. 5509-5522

7 Hanzo, L., Alamri, O., El-Hajjar, M., Wu, N.: 'Near-capacity multi-functional mimo systems' (Wiley-IEEE Press, 2009)

8 Ben Letaief, K., Zhang, W.: 'Cooperative communications for cognitive radio networks', Proc. IEEE, 2009, 97, pp. 878-893

$9 \mathrm{Li}, \mathrm{Y} .:$ 'Distributed coding for cooperative wireless networks: an overview and recent advances', IEEE Commun. Mag., 2009, 47, pp. $71-77$

10 Simeone, O., Gambini, J., Bar-Ness, Y., Spagnolini, U.: 'Cooperation and cognitive radio'. IEEE Int. Conf. Communications (ICC '07), June 2007, pp. 6511-6515

11 Su, W.F., Matyjas, J.D., Batalama, S.: 'Active cooperation between primary users and cognitive radio users in cognitive ad-hoc networks'. Proc. 2010 IEEE Int. Conf. Acoustics Speech and Signal Processing (ICASSP), March 2010, pp. 3174-3177

12 Su, W., Matyjas, J., Batalama, S.: 'Active cooperation between primary users and cognitive radio users in heterogeneous ad-hoc networks', IEEE Trans. Signal Process., 2012, 60, pp. 1796-1805

13 Han, Y., Pandharipande, A., Ting, S.: 'Cooperative decode-and-forward relaying for secondary spectrum access', IEEE Trans. Wirel. Commun., $2009, \mathbf{8}$, pp. $4945-4950$

14 Simeone, O., Stanojev, I., Savazzi, S., Bar-Ness, Y., Spagnolini, U., Pickholtz, R.: 'Spectrum leasing to cooperating secondary ad hoc networks', IEEE J. Sel. Areas Commun., 2008, 26, pp. 203-213

15 Bletsas, A., Khisti, A., Reed, D., Lippman, A.: 'A simple cooperative diversity method based on network path selection', IEEE J. Sel. Areas Commun., 2006, 24, pp. 659-672

$16 \mathrm{Ju}$, M., Kim, I.-M.: 'Relay selection with anc and tdbc protocols in bidirectional relay networks', IEEE Trans. Commun., 2010, 58, pp. $3500-3511$
$17 \mathrm{Ng}$, S.X., Hanzo, L.: 'On the MIMO channel capacity of multidimensional signal sets', IEEE Trans. Veh. Technol., 2006, 55, pp. $528-536$

18 Hanzo, L., Liew, T., Yeap, B., Tee, R., Ng, S.X.: 'Turbo coding, turbo equalisation and space-time coding for transmission over fading channels' (Wiley-IEEE Press, 2011)

19 Robertson, P., Wörz, T.: 'Bandwidth-efficient turbo trellis-coded modulation using punctured component codes', IEEE J. Sel. Areas Commun., 1998, 16, pp. 206-218

20 Ungerböck, G.: 'Channel coding with multilevel/phase signals', IEEE Trans. Inf. Theory, 1982, IT-28, pp. 55-67

21 Liu, K.J.R., Sadek, A.K., Su, W., Kwasinski, A.: 'Cooperative communications and networking' (Cambridge University Press, New York, 2009)

22 Goldsmith, A.: 'Wireless communication' (Cambridge University Press, England, 2005)

23 Tobagi Fouad, A., Hira, M.M.: 'Joint optimization of physical layer parameters and routing in wireless mesh networks'. Proc. Ninth IFIP Annual Mediterranean, Ad Hoc Networking Workshop (Med-Hoc-Net), 2010, June 2010, pp. 1-8

24 Kong, L.K., Ng, S.X., Maunder, R., Hanzo, L.: 'Maximum-throughput irregular distributed space-time code for near capacity cooperative communications', IEEE Trans. Veh. Technol., 2010, 59, pp. $1511-1517$

25 Ochiai, H., Mitran, P., Tarokh, V.: 'Design and analysis of collaborative diversity protocols for wireless sensor networks'. IEEE 60th, Vehicular Technology Conf., 2004 (VTC 2004) 2004, September 2004, vol. 7, pp. 4645-4649

26 Hanzo, L., Ng, S.X., Keller, T., Webb, W.: 'Quadrature amplitude modulation: from basics to adaptive trellis-coded, turbo-equalised and space-time coded OFDM, CDMA and MC-CDMA systems' (Wiley-IEEE Press, 2004)

27 Ng, S.X., Alamri, O., Li, Y., Kliewer, J., Hanzo, L.: 'Near-capacity turbo trellis coded modulation design based on EXIT charts and union bounds', IEEE Trans. Commun., 2008, 56, pp. 2030-2039

28 ten Brink, S.: 'Convergence behaviour of iteratively decoded parallel concatenated codes', IEEE Trans. Commun., 2001, 49, pp. 1727-1737

29 Kliewer, J., Ng, S.X., Hanzo, L.: 'Efficient computation of EXIT functions for non-binary iterative decoding', IEEE Trans. Commun., 2006, 54, pp. 2133-2136

30 Michalopoulos, D., Suraweera, H., Karagiannidis, G., Schober, R.: 'Amplify-and-forward relay selection with outdated channel estimates', IEEE Trans. Commun., 2012, 60, pp. 1278-1290

$31 \mathrm{Li}, \quad$ G., Xu, Z., Xiong, C., et al.: 'Energy-efficient wireless communications: tutorial, survey, and open issues', IEEE Wirel. Commun., 2011, 18, pp. 28-35

32 Duff, I.S., Erisman, A.M., Reid, J.K.: 'Direct methods for sparse matrices (Monographs on numerical analysis)' (Clarendon. Oxford University Press, Oxford, 1986) 


\section{www.ietdl.org}

\section{COMSI20130130}

1455 Author Queries

Wei Liang, Soon Xin Ng, Lajos Hanzo

Q1 Abstract should not be more than 200 words as per style. Please check and change.

Q2 Please expand PSK/QAM.

Q3 All footnotes have been moved to text as required by journal style. Please check and confirm that they have been located correctly within the text.

Q4 IEE style for matrices and vectors is to use bold italics. Please check that we have identified all instances. 